\title{
基于辐射定标的像元级双增益红外图像重构
}

\author{
行麦玲，杨小乐，邓旭光，杨天远
}

(北京空间机电研究所, 北京 100094)

\begin{abstract}
摘要: 非均匀性校正精度是空间红外相机图像质量的一项重要指标, 采用像元级双增益时间延迟积分 (Time Delay and Integration, TDI) 红外探测器得到的图像, 其校正精度与图像数据重构之后的线性度 直接相关。分析了红外 TDI 探测器像元级双增益成像时探测器输出信号的特点, 在此基础上提出基于 辐射定标的方法, 精确得到探测器每个像元高低增益输出值之间的等量关系, 确定每个像元的数据重 构系数, 提高重构之后的全动态范围内探测器信号的线性度, 从而提高红外图像非均匀性校正精度。 实验室测试数据验证结果表明, 基于辐射定标的高精度线性重构方法, 将红外图像的非均匀性校正精 度由 $4.1 \%$ 提高到 $1.2 \%$ 。
\end{abstract}

关键词: 红外成像; 像元级; 双增益图像重构

中图分类号：TP732.2 文献标识码：A 文章编号：1001-8891(2020)07-0670-06

\section{Pixel-Level Dual-Gain Infrared Image Reconstruction Based on Radiance Calibration}

\author{
XING Mailing, YANG Xiaole, DENG Xuguang, YANG Tianyuan \\ (Beijing Institute of Space Mechanics \& Electricity, Beijing 100094, China)
}

\begin{abstract}
Nonuniformity is a key indicator of the image data quality of a remote infrared sensor. The uniformity correction residual of the image obtained by a pixel-level, dual-gain, time delayed and integration(TDI) detector is closely related to the linearity after reconstruction. In this study, the signal readout process of the infrared TDI detector was analyzed. A new image data reconstruction method is presented to obtain accurate normal parameters for each pixel. The detector output linearity was increased in all dynamic ranges, and the uniformity of the image was enhanced. Radiance calibration was performed, and the test data were processed. The result shows that the nonuniformity correction residual decreased from $4.1 \%$ to $1.2 \%$ based on radiance calibration.
\end{abstract}

Key words: infrared imaging, pixel-level, reconstruction of dual-gain data

\section{0 引言}

在空间红外目标探测应用中, 大动态范围高灵敏 度探测与目标高精度检测是红外相机需要重点解决 的两方面问题: 首先以大动态范围高灵敏度探测能力 获取目标图像数据, 保证弱目标有足够的信噪比, 强 目标不饱和, 然后对图像进行非均匀性校正, 非均匀 性校正精度是实现高检测概率、低虚警率的前提 ${ }^{[1-4]}$ 。 在相机成像过程中, 远距离弱目标在探测器焦面上形 成的辐射量非常小, 要保证一定的探测信噪比, 要求 相机灵敏度极高, 通常会采用 TDI 结合小电容读出的 方式以增大响应电压, 提高信噪比。然而小电容的满 阱电子数比较小, 在同一时刻出现近距离强目标时,
容易饱和, 导致无法测量其辐射量。常规的解决办法 是, 在强目标信号出现时, 相机通常采取长短积分时 间调节和大小电容调节减小信号累积 ${ }^{[5-9]}$, 以避免探测 器饱和。这两种方式都是将探测器所有像元同时改变 其积分时间和电容, 如此以来, 在强弱目标共存时, 无法保证弱目标的有效探测。洪闻青等人 ${ }^{[10]}$ 提出采用 基于不同积分时间帧累加的红外图像超帧方法在一 定程度上可以同时兼顾灵敏度和动态范围两方面性 能, 但由于需要采集存储多个子帧数据, 数据量缓存 要求高, 不利于空间相机星上实时处理。为此像元级 双增益自动控制读出电路的探测器应运而生 ${ }^{[11]}$ 。采用 这种方式的 TDI 探测器, 其每列像元均有独立的高低 增益自动控制成像能力。 
双增益自动控制探测器的输出信号对入射辐射 能量的响应不单调, 非均匀性校正前必须先做增益归 一化 ${ }^{[12-13]}$, 完成图像数据重构。重构方法将影响非均 匀性校正精度, 从而影响目标检测概率和虚警率。文 献[5]中双增益数据重构采用了固定参数的方法, 即所 有像元采用同一组参数归一化。由于像元级双增益探 测器每个像元的读出电路配置无法做到完全一致, 像 元间的增益有所不同, 因此, 这种方法对像元级双增 益数据适应性较差。

文献[11]在读出电路硬件设计上采用了多电容采 样并结合独特的内部处理方式, 可以去除像元间增益 差异导致的非均匀性, 但这种方式对读出电路的要求 很高, 使读出电路复杂化, 实现难度大, 极大地限制 了像元级双增益探测器的开发与应用。

本文分析了红外 TDI 探测器像元级双增益自动 控制成像及其信号响应原理, 提出基于辐射定标的图 像数据重构方法, 在不增加读出电路复杂度的情况 下, 提高图像非均匀性校正精度。开展了辐射定标实 验, 通过实验数据验证了基于辐射定标法重构的非均 匀性校正精度。这种方法用于大规模像元级双增益红 外探测器时, 能够降低读出电路研发难度, 提高其可 靠性, 满足空间红外目标大动态范围高灵敏度探测需 求。

\section{1 像元级双增益自动控制成像原理}

下面以 1024 列 5 元参与 TDI 的短波红外探测器 为例做介绍。

探测器读出电路中每列像元通道均设计有比较 器, 比较器设定阈值电压。相机在扫描成像过程中, TDI 探测器每列像元的 5 个元参与 TDI 转移和信号累 加。累加输出后的信号电压值与阈值电压比较, 若小 于國值电压, 则输出 5 元累加后的信号值, 表现为高 增益成像, 否则, 仅输出中间元的信号, 表现为低增
益成像。在此过程中, 中间元的信号分别保存于两个 存储电容, 其中一个电容的信号用于参与 TDI 信号累 加, 实现相机高增益成像, 另一个电容的信号用于单 独输出, 实现低增益成像。

由于每列像元都有独立的比较器, 因此, 实现了 像元级双增益自动控制成像。

高增益输出信号还可进一步通过放大器调节增 益倍数, 针对不同应用需求设置不同倍数。

\section{2 基于辐射定标的数据重构方法}

在红外相机系统成像时, 探测器每个像元的输出 电压由入瞳辐射形成的电压、光机辐射形成的电压、 暗电流形成的电压, 以及读出电路本底电压 4 部分组 成。

探测器对入瞳辐射响应形成的电压记为 $U_{\mathrm{Enp}}$, 则:

$$
U_{\text {Enp }}=L_{\text {Enp }} \tau_{\mathrm{o}} \Omega_{\mathrm{F}} A_{\mathrm{d}} \tau_{\text {int }} \frac{\lambda}{h c} \eta \frac{Q_{\mathrm{e}}}{C_{0}}
$$

式中: $L_{\mathrm{Enp}}$ 为入瞳辐射亮度; $\tau_{0}$ 为光学系统效率; $\Omega_{\mathrm{F}}$ 为成像立体角 (即光学系统相对孔径形成的像方立体 角); $A_{\mathrm{d}}$ 为探测器像元面积; $\tau_{\mathrm{int}}$ 为积分时间; $\lambda$ 为探 测器响应波长; $h$ 为普朗克常数; $c$ 为光速; $\eta$ 为量子 效率; $Q_{\mathrm{e}}$ 为电荷电量, $1.6 \times 10^{19} \mathrm{C} ; C$ 为探测器电容。 探测器对光机辐射响应形成的电压记为 $U_{\mathrm{Om}}$, 则:

$$
U_{\mathrm{Om}}=L_{\mathrm{Om}} \Omega_{\mathrm{Om}} A_{\mathrm{d}} \tau_{\mathrm{int}} \frac{\lambda}{h c} \eta \frac{Q_{\mathrm{e}}}{C}
$$

式中: $L_{\mathrm{Om}}$ 为光机辐射对探测器形成的光谱辐亮度;

$\Omega_{\mathrm{Om}}$ 为探测器焦面接收光机辐射的立体角。

由探测器暗电流形成的电压记为 $U_{\mathrm{Dc}}$, 则:

$$
U_{\mathrm{Dc}}=I_{\mathrm{Dc}} \tau_{\mathrm{int}} Q_{\mathrm{e}} / C
$$

式中: $I_{\mathrm{Dc}}$ 为暗电流。

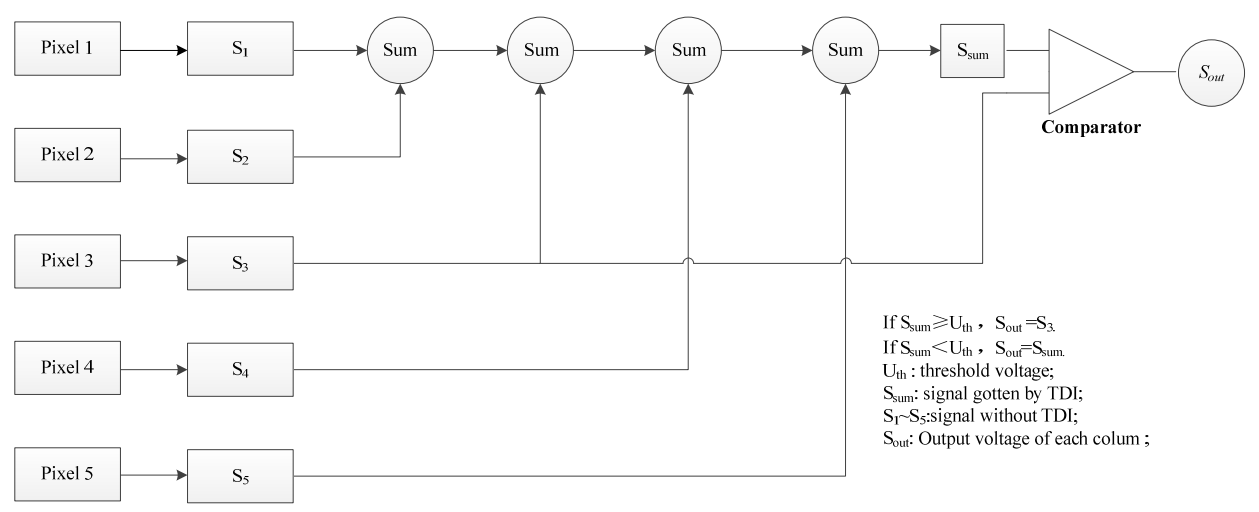

图 1 像元级双增益探测器信号读出原理示意图

Fig.1 Pixel-level dual-gain detector signal readout schematic diagram 
由图 1 和式(1) (3)可以看出, $U_{\mathrm{Enp}} 、 U_{\mathrm{Om}}$ 和 $U_{\mathrm{DC}}$ 在同一列每个参与 TDI 的像元输出值进行累加时, 均 有等量贡献。因此在 TDI 多元累加输出的高增益模式 和 1 元输出的低增益模式下, 探测器输出的总电压可 写为如下形式:

$$
\begin{gathered}
U_{\mathrm{Hg}}=\left(U_{\mathrm{Enp}}+U_{\mathrm{Om}}+U_{\mathrm{Dc}}\right) f_{\mathrm{g}}+U_{\mathrm{OH}} \\
U_{\mathrm{Lg}}=\left(U_{\mathrm{Enp}}+U_{\mathrm{Om}}+U_{\mathrm{Dc}}\right)+U_{\mathrm{OL}}
\end{gathered}
$$

式中: $U_{\mathrm{Hg}} 、 U_{\mathrm{Lg}}$ 分别为探测器高增益和低增益的输出 电压值; $f_{\mathrm{g}}$ 为高低增益之间的倍数; $U_{\mathrm{OH}} 、 U_{\mathrm{OL}}$ 分别为 高增益和低增益输出时的读出电路本底电压。

由式(1) (5)可得到, 在只改变相机入瞳辐射量的 情况下, 探测器输出电压与相机入瞳辐射之间的关系 可表示为:

$$
\begin{aligned}
& U_{\mathrm{Hg}}=L_{\mathrm{Enp}} R f_{\mathrm{g}}+\left(U_{\mathrm{Om}}+U_{\mathrm{Dc}}\right) f_{\mathrm{g}}+U_{\mathrm{OH}} \\
& U_{\mathrm{Lg}}=L_{\mathrm{Enp}} R+\left(U_{\mathrm{Om}}+U_{\mathrm{Dc}}\right)+U_{\mathrm{OL}}
\end{aligned}
$$

式中: $R$ 为探测器低增益输出的响应率。将式(6)中与 入瞳辐射无关的量合并记为 $U_{0}$, 式(7)中与入睲辐射 无关的量合并记为 $U_{0}{ }^{\prime}$, 则:

$$
\begin{aligned}
& U_{\mathrm{Hg}}=L_{\mathrm{Enp}} R f_{\mathrm{g}}+U_{0} \\
& U_{\mathrm{Lg}}=L_{\mathrm{Enp}} R+U_{0}^{\prime}
\end{aligned}
$$

式(8)、(9)分别描述了探测器每列像元高增益和低 增益输出时, 探测器输出电压随相机入瞳辐射亮度的 变化关系。由此可见, 对于相机来说, 通过辐射定标 的方法可以确定 $R 、 f_{\mathrm{g}} 、 U_{0}$ 和 $U_{0}{ }^{\prime}$ 。然后由式(8)、(9), 可得每个像元输出电压的重构方程为:

$$
U_{\text {nor }}= \begin{cases}U \frac{1}{f_{\mathrm{g}}}+\left(U_{0}^{\prime}-\frac{1}{f_{\mathrm{g}}} U_{0}\right), & U<U_{\text {th }} \\ U, & U \geq U_{\text {th }}\end{cases}
$$

式中: $U_{\mathrm{th}}$ 为比较器阈值电压; $\left(U_{0}^{\prime}-\frac{1}{f_{\mathrm{g}}} U_{0}\right)$ 为重构 以后的偏置系数。由重构算法可知, 重构时高增益输 出电压值按照增益倍数和偏置系数做线性变换, 低增 益输出值保持不变。

\section{3 辐射标定实验与数据重构}

为验证上述模型的准确性, 在实验室环境下 (控 温 $20^{\circ} \mathrm{C} \pm 2^{\circ} \mathrm{C}$ ), 对具有像元级双增益的 $1024 \times 5$ 短 波红外 TDI 探测器开展了辐射定标实验。如图 2 所示, 将黑体放置在探测器入光口前, 距离探测器组件入光 口 $10 \mathrm{~mm}$, 能够覆盖探测器冷屏光阑和全部像元。

实验所用黑体及探测器组件的主要性能参数见 表 1 和表 2 。
实验中, 用黑体辐射等效入瞳处辐亮度经光学系 统之后到达探测器冷光阑处的辐亮度, 通过改变黑体 温度来改变辐亮度, 从而改变探测器光敏面上的辐照 度。在探测器响应动态范围内, 在 $283 \mathrm{~K} \sim 446 \mathrm{~K}$ 范围 内标定了 30 个温度点, 获得了不同辐亮度输入时探 测器的输出信号电压值。选取第 4 像元和第 5 像元进 行分析, 这两个像元分别代表奇元和偶元的响应情 况。在双增益模式下, 第 4 像元和第 5 像元的响应输 出与辐亮度值见图 3。

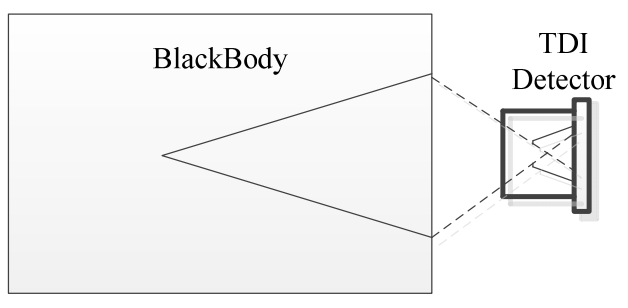

图 2 探测器实验室辐射定标

Fig.2 Radiance calibration of detector in lab 表 1 黑体主要性能参数

Table 1 Performance of the blackbody

\begin{tabular}{lc}
\hline \multicolumn{1}{c}{ Performance } & Value \\
\hline Irradiance temperature range & $280 \mathrm{~K}-500 \mathrm{~K}$ \\
Temperature measurement error & $0.01 \mathrm{~K}$ \\
Emissivity & 0.999 \\
Temperature stability & $0.01 \mathrm{~K}$ \\
Thermal uniformity & $0.01 \mathrm{~K}$ \\
Emissive area & $\phi 50 \mathrm{~mm}$ \\
\hline
\end{tabular}

表 2 探测器主要性能参数

Table 2 Performance of detector subassembly

\begin{tabular}{ll}
\hline Performance & Value \\
\hline Spectrum & $2.8-3 \mu \mathrm{m}$ \\
Pixel array size & 1024 \\
Pitch & $15 \mu \mathrm{m}$ \\
F/\# & 2 \\
Cold shield diameter & $40 \mathrm{~mm}$ \\
Reponsivity uniform & $8 \%$ \\
Gain designed & 5 \\
\hline
\end{tabular}

由图 3 数据点可以看出, 奇偶元之间的差异较为 明显, 这是 TDI 探测器奇偶元响应特点之一。全部测 点的响应输出明显表现为线性阶跃, 由大斜率向小斜 率变化时, 即表明随着黑体温度升高, 辐亮度增大, 高增益响应输出值达到饱和, 大于比较器阈值, 探测 器以低增益输出, 像元增益发生了自动切换。

同一像元的高增益与低增益电压输出值的斜率 和偏置不同。不同像元的增益切换点、斜率和偏置也 有所不同, 这也是影响探测器非均匀性的因素之一, 数据重构需考虑这一点。 


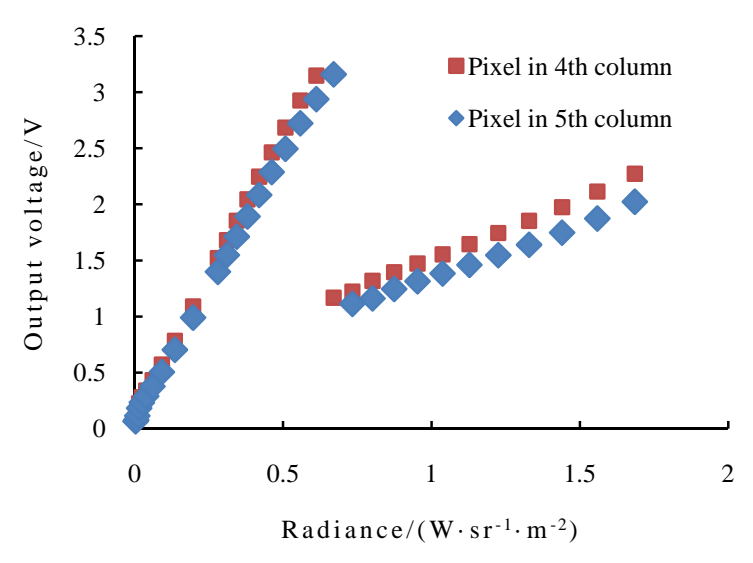

图 3 两列像元的双增益输出值

Fig.3 Dual-gain output of two column pixels

下面比较两种重构方法: 固定参数法和辐射定标 法。

\section{1 基于固定参数法的重构结果}

固定参数法是目前自动增益成像数据重构的常 规方法, 即根据电路设计结果确定高低增益倍数和偏 置系数，对所有元输出值采用相同参数进行重构。

根据电路参数设计值, 实验测试用探测器的高低 增益倍数设计值 $f_{\mathrm{g}}$ 为 5.3 , 偏置系数为 $0.5 \mathrm{~V}$ 。采用此 参数对所有像元重构, 其中第 4 像元和第 5 像元重构 结果见图 4。

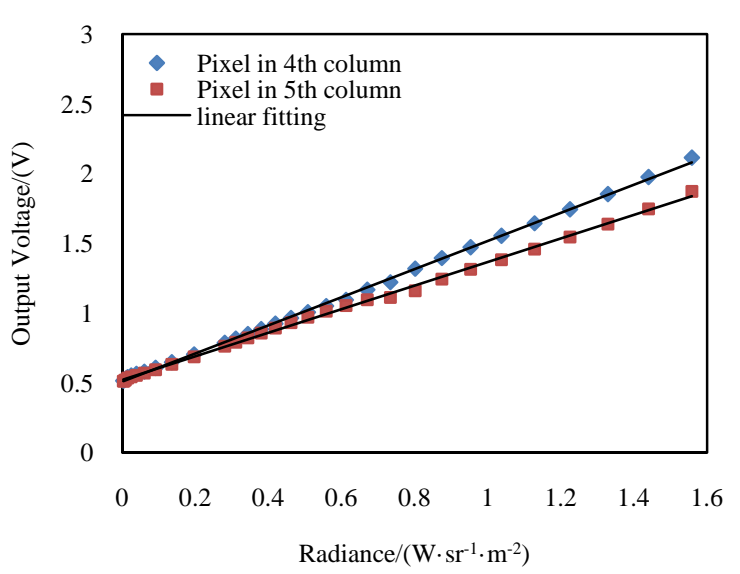

图 4 采用固定参数法重构的两列像元输出值

Fig.4 Reconstruction results of two pixels based on fixed parameters

重构之后的数据在增益切换前后仍然有一定阶 跃, 尤其是像元 5 较为明显。采用最小二乘法线性拟 合得到两个像元各自的重构方程为:

$$
\left\{\begin{array}{l}
U_{4, \text { nor }}=1.0085 L_{4, \text { Enp }}+0.5092 \\
U_{5, \text { nor }}=0.8448 L_{5, \text { Enp }}+0.522
\end{array}\right.
$$

第 4 像元的输出电压值与入睲辐亮度的线性相关 系数大于 0.9992 , 第 5 像元的输出电压值与入瞳辐亮 度的线性相关系数大于 0.9985 。

\section{2 基于辐射定标法的重构结果}

采用辐射定标法确定像元 4 的增益倍数为 5.16, 偏置系数为 0.51 。像元 5 的增益倍数为 5.66 , 偏置系 数为 0.49 。

按照式(11), 第 4 像元和第 5 像元的输出值重构 结果见图 5。

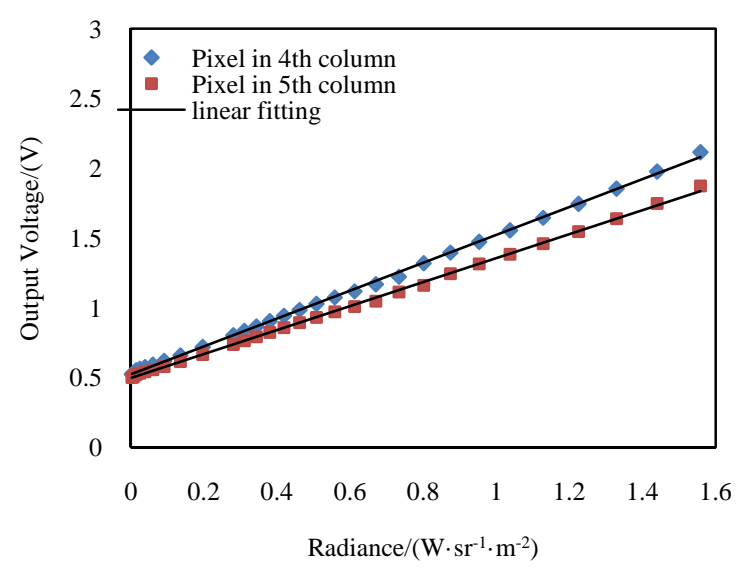

图 5 采用辐射定标方法重构某两列像元输出

Fig.5 Reconstruction results of two pixels based on radiance calibration

采用最小二乘法线性拟合图 5 数据, 得到两个像 元各自的重构方程为:

$$
\left\{\begin{array}{l}
U_{4, \text { nor }}=0.9993 L_{4, \text { Enp }}+0.5229 \\
U_{5, \text { nor }}=0.8594 L_{5, \text { Enp }}+0.4965
\end{array}\right.
$$

第 4 像元的输出电压值与入瞳辐亮度的线性相关 系数大于 0.9994 , 第 5 像元的输出电压值与入瞳辐亮 度的线性相关系数大于 $0.9992 . 。$ 辐射定标法重构方程 的线性相关系数有所提高, 输出电压值也看不出阶 跃。

\section{4 精度分析}

\section{1 精度影响因素分析}

实验过程中可能影响标定精度的因素如下:

1）黑体测温精度：会影响增益重构精度，最高 标定点辐亮度为 $1.685 \mathrm{~W} /\left(\mathrm{sr} \cdot \mathrm{m}^{2}\right)$, 测温精度 $0.01 \mathrm{~K}$, 对 标定精度的影响为 $0.05 \%$;

2）黑体均匀性：会造成焦面照度不均匀，直接 影响非均匀性校正精度,对标定精度的影响为 $0.05 \%$;

3) 探测器冷屏: 在焦面处会造成照度不均匀, 影响非均匀性校正精度, 对标定精度的影响为 $0.05 \%$; 
4) 黑体发射率: 影响增益重构精度, 发射率不 确定度为 0.001 , 对标定精度的影响为 $0.1 \%$;

5）黑体稳定度: 影响增益重构精度, 稳定度 $0.01 \mathrm{~K}$ ，对标定精度的影响为 $0.05 \%$;

6) 探测器噪声: 影响增益重构和非均匀性校正 精度, 噪声 $0.7 \mathrm{mV}$, 对标定精度的影响为 $0.04 \%$;

上述各因素之间不相关, 因此, 取其均方根作为 对精度的综合影响结果，经计算为 $0.52 \%$ 。

\section{2 两种方法精度对比}

首先分析两种不同方法重构之后的线性度差异。 为了具体分析全动态范围内线性情况, 通过所有点的 拟合残差比较线性度。选择重构后阶跃较为明显的像 元 5 做对比, 两种重构方法的拟合残差见图 6。

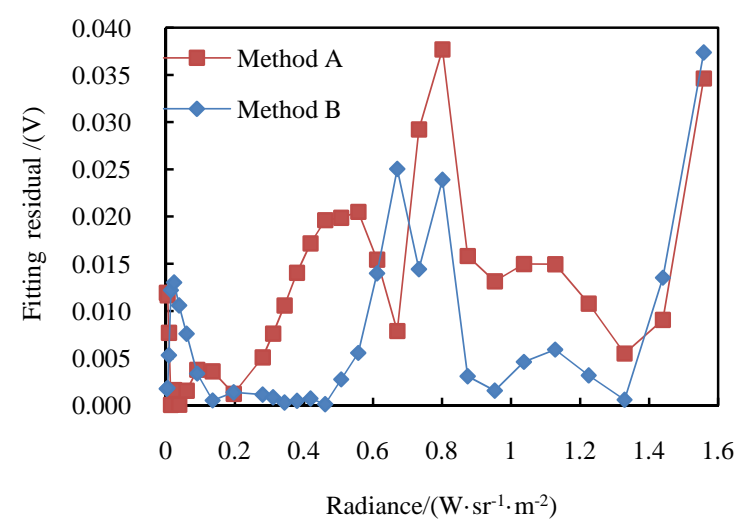

图 6 两种重构数据的线性拟合残差（方法 A: 固定参数法; 方法 B: 辐射定标法)

Fig.6 Linear fitting residual by two methods(method A: fixed parameters. method B: radiance calibration)

由图 6 可见, 虽然两种方法的最大拟合残差相当, 但是基于辐射定标法重构数据的线性拟合残差整体 小于基于固定参数法, 而且基于辐射定标法的最大拟 合残差出现在即将饱和的区域。因此, 实际成像并做 辐射量反演时, 基于辐射定标法重构数据的使用价值 更好。双增益输出值重构后的线性度对非均匀性校正 精度产生直接影响, 进一步比较两种方法重构数据的 非均匀性校正精度。

对 1024 列像元做非均匀性定标, 以 281.68K (谱 段辐亮度为 $0.002625 \mathrm{~W} /\left(\mathrm{sr} \cdot \mathrm{m}^{2}\right.$ ) 和 $420.8 \mathrm{~K}$ (谱段辐亮度 为 $0.874301 \mathrm{~W} /\left(\mathrm{sr} \cdot \mathrm{m}^{2}\right)$ 两点作为非均匀性定标温度点, 计算校正系数, 并计算其它各温度点下校正精度。

两点法校正探测器像元之间非均匀性校正算法 如下:

$$
\overline{U_{\mathrm{H}}}=k_{i} U_{i, \mathrm{H}}+b_{i}
$$

$$
\overline{U_{\mathrm{L}}}=k_{i} U_{i, \mathrm{~L}}+b_{i}
$$

式中: $U_{i, \mathrm{H}} 、 U_{i, \mathrm{~L}}$ 分别为高温定标和低温定标时, 像元 $i$ 的图像输出值; $\overline{U_{\mathrm{H}}}$ 为高温定标时, 所有像元的图像 输出均值; $\overline{U_{\mathrm{L}}}$ 为低温定标时, 所有像元的图像输出 均值; $k_{i}$ 和 $b_{i}$ 为像元 $i$ 的非均匀性校正系数。

由式(13)、式(14)可得到 $k_{i}$ 和 $b_{i}$ 为:

$$
\begin{gathered}
k_{i}=\frac{\overline{U_{\mathrm{H}}}-\overline{U_{\mathrm{L}}}}{U_{i, \mathrm{H}}-U_{i, \mathrm{~L}}} \\
b_{i}=\overline{U_{\mathrm{H}}}-k_{i} U_{i, \mathrm{H}} \\
U_{i}^{\prime}=k_{i} U_{i}+b_{i}
\end{gathered}
$$

式(17)中: $U_{i}^{\prime}$ 为像元 $i$ 校正后的图像输出值; $U_{i}$ 为像 元 $i$ 校正前的图像输出值。对图像数据先重构, 之后 按照式(15)、式(16)计算各像元的非均匀性校正系数, 然后按照式(17)进行非均匀性校正。

全线列像元两种重构方法在各温度点下的非均 匀性校正精度见图 7 数据。

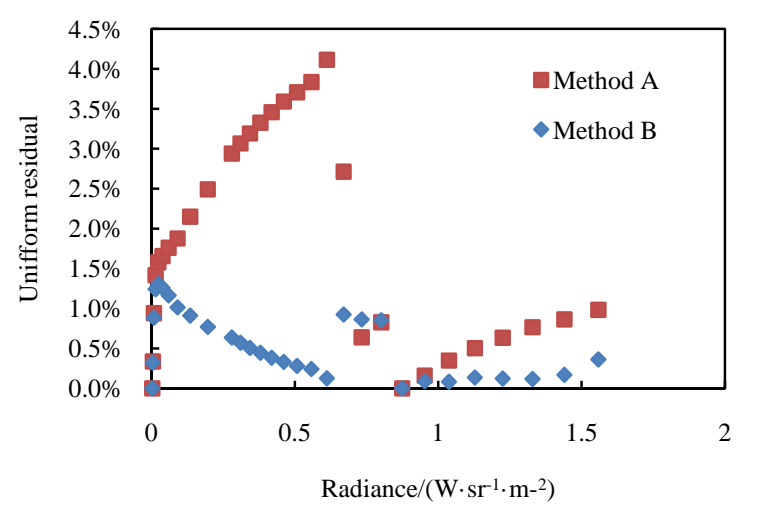

图 7 两种方法重构数据的非均匀性校正精度 (方法 A: 固定 参数法; 方法 B：辐射定标法)

Fig.7 Non-uniformity after correction by two methods(method

A: fixed parameters, method B:radiance calibration)

由非均匀性校正精度比对可知, 基于辐射定标法 重构数据的全部测点校正精度整体明显优于固定参 数法。基于固定参数法重构数据的非均匀性校正精度 最低为 $4.1 \%$, 而基于辐射定标法重构数据的非均匀性 校正精度最低为 $1.2 \%$ 。可见, 基于辐射定标法重构双 增益图像数据, 可明显提高非均匀性校正精度。 


\section{5 结论}

针对像元级双增益红外 TDI 探测器输出信号特 点, 提出了基于辐射定标法的图像数据重构方法, 通 过对辐射定标实验数据进行线性拟合得到各像元的重 构方程。实验结果表明, 基于辐射定标法重构数据, 将测试探测器输出电压值的非均匀性校正精度由 $4.1 \%$ 提高到了 $1.2 \%$ 。在不增加读出电路复杂度的情况下, 该方法改善了像元级双增益成像的图像质量, 这一点 对于降低红外目标检测虚警率非常有意义, 可应用于 大动态范围高精度红外目标探测相机及其目标检测 系统。

\section{参考文献:}

[1] 汪江华, 于素芬, 李国强, 等. 焦平面探测器图像非均匀性对目标检 测的影响 [J]. 电光与控制, 2010, 17(10):72-74.

WANG Jianghua, YU Sufen, LI Guoqiang, et al. Effect of Image Nonuniformity of Focal Plane Detector on Target Detection[J]. Electronics Optics \& Control, 2010, 17(10): 72-74.

[2] 赵明, 林长青. 基于 N-P 准则的红外线列非均匀性对目标检测的影响 分析[J]. 红外技术, 2012, 34(4): 237-242.

ZHAO Ming, LIN Changqing. Influence Analysis of Non-uniformity of Infrared Line Array Detector for Target Detection based on Neyman-Person Criterion[J]. Infrared Technology, 2012, 34(4): 237-242.

[3] 高国旺. 全向 IRST 系统的图像处理与信息融合技术研究[D]. 西安: 西安电子科技大学, 2013: 25-31.

GAO Guowang. Research on Infrared Image Processing and InformationFusion Technology of Omni-direction IRST System[D]. Xi’an: XiDian University,2013:25-31.

[4] 张丽莎, 刘兆军, 马文坡, 等. 基于 $90^{\circ}$ 旋转定标和场景校正相结合的 非均匀性校正技术[J]. 航天返回与遥感, 2017, 38(1): 78-87.

ZHANG Lisha, LIU Zhaojun, MA Wenpo, et al. High Performance NUC by Side-slither Combined with Scened-based Correction[J]. Spacecraft Recovery \& Remote Sensing, 2017, 38(1):78-87.

[5] 韩建强, 黄北举, 关宁, 等. 一种高灵敏度大动态范围读出电路[J]. 中 国集成电路, 2011, 20(1): 39-42.

HAN Jianqiang, HUANG Beiju, GUAN Ning, et al. A high sensitivity and wide dynamic range readout circuit[J]. China Integrated Circuit, 2011, 20(1): 39-42.
[6] 张大纬, 李江勇. 用于红外成像系统的自动增益控制方法研究 [J]. 激 光与红外, 2009, 39(10): 1119-1122.

ZHANG Dawei, LI Jiangyong. Research of the automatic-gain control method for infrared imaging system[J]. Laser \& Infrared, 2009, 39(10): 1119-1122.

[7] 张恒伟, 冀翔, 赵威. 激光对红外热成像设备自动增益控制电路的干 扰效果[J]. 强激光与粒子束, 2011, 23(9): 2325-2328.

ZHANG Hengwei, JI Xiang, ZHAO Wei. Jamming effect of laser on infrared thermal imager's automatic gain control circuit[J]. High Power Laser and Particle, 2011, 23(9): 2325-2328.

[8] 张丽莎, 刘兆军, 马文坡, 等. 像素级数字积分红外成像技术研究 [J]. 激光与光电子学进展, 2016, 53(9): 091102-1-091102-8.

ZHANG Lisha, LIU Zhaojun, MA Wenpo, et al. Infrared Imaging Technology Based on Pixel-Level Digital Integration[J]. Laser \& Optoelectronics Progress, 2016, 53(9) : 091102-1-091102-8.

[9] 彭妮娜, 陈大羽, 王琨, 等. 采用线阵 TDICCD 相机的实时自动增益 控制算法 [J]. 红外与激光工程, 2011, 40(7): 1370-1375.

PENG Nina, CHEN Dayu, WANG Kun, et al. Real-time automatic gain control algorithm based on linear TDICCD camera[J]. Infrared and Laser Engineering, 2011, 40(7): 1370-1375.

[10] 洪闻青, 姚立斌, 姬荣斌, 等. 基于不同积分时间帧累加的红外图像 超帧方法 [J]. 光学精密工程, 2016, 24(6): 1490-1500.

HONG Wenqing, YAO Libin, JI Rongbin. A super-frame processing method for infrared image based on accumulation of different integration time frame[J]. Optics and Precision Engineering, 2016, 24(6): 1490-1500.

[11] Pain Bedabrata. Increasing Linear Dynamic Range of a CMOS Image Sensor[R]. NASA Tech Briefs, 2007: 7-8.

[12] 钟胜, 张天序, 桑农, 等. 从因 AGC 受损的红外图像中计算点源能 量比[J]. 系统工程与电子技术, 2003, 25(9): 1113-1115.

ZHONG Sheng, ZHANG Tianxu, SANG Nong, et al. Calculation of the Infrared Radiant Intensity Ratio of Point Sources from the Infrared Image Spoilt by AGC[J]. Systems engineering and electronics, 2003, 25(9): 1113-1115.

[13] Dong S K, Eun K, Choul W S. Noise Power Spectrum Measurements under NonuniformGains and Their Compensations[C]//Medical Imaging2016: Physics of Medical Imaging, Proc. of SPIE, 2016, 9783: 97833S-1-97833S-9. 